\title{
Induction of c-Fos and $\Delta$ FosB Immunoreactivity in Rat Brain by Vagal Nerve Stimulation
}

\author{
J Thomas Cunningham', Steven W Mifflin', Georgianna G Gould' and Alan Frazer*,' \\ 'Department of Pharmacology, University of Texas Health Science Center, San Antonio, TX, USA
}

\begin{abstract}
Vagus nerve stimulation (VNS) is used as therapy for treatment-resistant depression or epilepsy. This study used immunohistochemistry for biomarkers of short-term (c-Fos) and long-term ( $\Delta$ FosB) neuronal activation to map regions in brain that are activated by acute $(2 \mathrm{~h}$ ) or chronic ( 3 weeks) VNS in conscious Sprague-Dawley rats. Electrodes (Cyberonics Inc.) were implanted on the left vagus nerve and I week after surgery, stimulation began using parameters employed clinically (one burst of $20 \mathrm{~Hz}, 250 \mu$ s pulse width, $0.25 \mathrm{~mA}$ stimulation for $30 \mathrm{~s}$ every $5 \mathrm{~min}$ ). Radio telemetry transmitters were used for monitoring blood pressure, heart rate, activity, and respiratory rate during VNS; neither acute nor chronic VNS significantly affected these parameters. Acute VNS significantly increased c-Fos staining in the nucleus of the solitary tract, paraventricular nucleus of the hypothalamus, parabrachial nucleus, ventral bed nucleus of the stria terminalis, and locus coeruleus but not in the cingulate cortex or dorsal raphe nucleus (DRN). Acute VNS did not affect $\Delta$ FosB staining in any region. Chronic VNS significantly increased $\Delta$ FosB and c-Fos staining bilaterally in each region affected by acute VNS as well as in the cingulate cortex and DRN. Using these stimulation parameters, VNS was tested for antidepressant-like activity using the forced swim test (FST). Both VNS and desipramine significantly decreased immobility in the FST; whereas desipramine decreased immobility by increasing climbing behavior, VNS did so by increasing swimming behavior. This study, then, identified potential sites in brain where VNS may produce its clinical effects.

Neuropsychopharmacology (2008) 33, I884- |895; doi: 10. 1038/sj.npp. I 30 1570; published online 24 October 2007
\end{abstract}

Keywords: vagal nerve stimulation; c-Fos; $\Delta$ FosB; forced swim test; depression

\section{INTRODUCTION}

Vagal nerve stimulation (VNS) has been approved by the Food and Drug Administration for treatment of refractory epilepsy (1997) and more recently (2005) for treatment of refractory depression. With respect to efficacy in depression, a 12-week study comparing severely ill patients who received VNS in addition to the medications they had been receiving did not improve significantly more than those who received sham treatment (Rush et al, 2005a). However, follow-up of those patients who had received VNS and continued receiving such stimulation for an additional 9 months or switching the sham-treated patients to VNS for 12 months indicated a time-dependent increase in efficacy (Rush et al, 2005b). After 12 months of VNS, both response and remission rates were about double those at 3 months. In this cohort, it was found that if patients responded beneficially to VNS after either 3 or 12 months of treatment,

\footnotetext{
*Correspondence: Dr A Frazer, Department of Pharmacology, University of Texas Health Science Center, 7703 Floyd Curl Drive, Mail Code 7764, San Antonio, TX 78229-3900, USA,

Tel: + I 210567 4202, Fax: + I 2105674300 ,

E-mail: frazer@uthscsa.edu
}

Received 18 July 2007; revised 9 August 2007; accepted 9 August 2007 then efficacy was maintained for up to 24 months (Sackeim et al, 2007).

How VNS produces its beneficial clinical effects is unknown. The vagus is a mixed nerve with $80 \%$ of the fibers carrying afferent sensory information to the central nervous system (CNS) (Foley and DuBois, 1937; Nemeroff et al, 2006). The cell bodies of the afferent fibers are located in the nodose ganglia and project to the nucleus of the solitary tract (NTS). The NTS then sends direct projections to many regions, including cell bodies for noradrenergic or serotonergic neurons, namely the locus coeruleus (LC) and dorsal raphe nucleus (DRN), respectively (Groves and Brown, 2005; Nemeroff et al, 2006), with such neurons thought to be involved in the mechanisms of action of antidepressants. In addition to these direct projections, the NTS sends indirect projections widely throughout the brain (Groves and Brown, 2005; Nemeroff et al, 2006). Human imaging studies carried out with VNS also show widespread effects on subcortical and cortical regions (Barnes et al, 2003; Henry et al, 2004, 1998). Thus, stimulation of the vagus would be expected to cause widespread effects in many areas of brain.

Several studies have examined the ability of VNS to 'activate' brain regions using increases in Fos protein (Curran and Morgan, 1995; Herdegen and Leah, 1998; Senba and Ueyama, 1997) as the measure of activation (Gieroba 
and Blessing, 1994; Naritoku et al, 1995; Osharina et al, 2006; Rutherfurd et al, 1992; Yousfi-Malki and Puizillout, 1994). All these studies measured the ability of acute VNS, for 30-180 min, to increase Fos protein in brain of either rats or rabbits. In all but one study (Gieroba and Blessing, 1994), stimulation was carried out in anesthetized animals. Anesthesia can increase Fos protein throughout brain (Takayama et al, 1994), which could mask effects of vagal stimulation. Anesthesia can also change the threshold for activation of different types of fibers in the vagal bundle (Woodbury and Woodbury, 1990). Further, in all but one study (Naritoku et al, 1995), stimulation of the vagus was carried out using either electrodes or stimulation parameters that resulted in alterations of peripheral autonomic function, (eg mean arterial pressure, MAP; heart rate, HR; and/or respiratory frequency, RF). Such changes would be expected to produce reflexes that could activate brain regions thereby complicating the interpretation of the results.

In view of these considerations, it seemed worthwhile to reexamine this issue using clinically relevant stimulation parameters in conscious rats. Also, given the long-term nature of VNS, it also appeared useful to examine effects of VNS after more chronic stimulation. Consequently, in nonanesthetized rats we studied the effects of both acute $(2 \mathrm{~h})$ or more chronic ( 3 weeks) VNS using c-Fos or $\Delta F o s B$ as markers of activation. Fos is an immediate early gene product and has been used to indicate acute activation of cells, usually peaking within $1-3 \mathrm{~h}$ of stimulus exposure (Kovacs, 1998). By contrast, FosB and its splice variant $\Delta$ FosB show a more delayed activation but persist longer than c-Fos; consequently, they have been suggested to be markers of chronic neuronal activation (Nestler, 2004).

\section{MATERIALS AND METHODS}

\section{Animals}

Experiments were carried out using adult male SpragueDawley rats, 250-350 g (Charles River). Rats were individually housed and maintained in a temperaturecontrolled environment on a $14: 10 \mathrm{~h}$ light-dark cycle. Rats had ad libitum access to food and water. Experimental protocols were approved by the IACUC in accordance with the guidelines of the Public Health Service, American Physiological Society, and Society for Neuroscience.

Vagus nerve electrodes were implanted on the left vagus nerve under aseptic conditions. The surgical procedure was similar to that described by Dorr and Debonnel (2006) except that the anesthetic was $2 \%$ isofluorane. Electrodes were connected to a stimulator pack that was sutured in and placed in a subcutaneous pouch created on the back of the rat. Both the electrode and stimulator were supplied by Cyberonics Inc. (Houston, TX). The bipolar stimulating electrode was configured with the cathode as the proximal lead and the anode at the distal lead to preferentially direct action potential propagation toward the CNS by creating anodal block at the distal lead. Rats that received VNS $(n=15)$ were instrumented with an operational stimulator pack that was programmed by a handheld computer.
Controls received a dummy simulator pack $(n=14)$ that was the same size and weight $(48 \mathrm{~mm} \times 33 \mathrm{~mm} \times 7.1 \mathrm{~mm}$; $16 \mathrm{~g})$.

\section{Telemetry}

Some rats were also instrumented with a radio telemetry transmitter (Data Sciences Instr.) so as to monitor systolic and diastolic blood pressure, HR, RF, and activity. Systolic and diastolic blood pressures were used to calculate an MAP that was used for statistical analysis. Physical activity was assessed by measuring changes in the animal's transmitter signal strength. When the animal changed position, the change in transmitter signal strength relative to the reference point was measured as an increase in counts/ min. Low counts indicated reduced physical activity in the animal. The activity data provided by this system is strictly a measure of locomotor activity and has been used widely for this purpose (Ansah et al, 1996; Howarth et al, 2005; Kawashima et al, 1996; Meerlo et al, 1999; Zhang et al, 2004). For the acute study, radio telemetry signals were recorded continuously from each individual at a rate of $64 \mathrm{~Hz}$. Signals were averaged during a $1 \mathrm{~h}$ baseline period before stimulation, during the first hour of VNS or sham stimulation, the second hour of VNS or sham stimulation, and for a $30 \mathrm{~min}$ poststimulation or sham recovery period. In the chronic study, radio telemetry signals were sampled continuously for $10 \mathrm{~s}$ every $10 \mathrm{~min}, 24 \mathrm{~h}$ a day for the duration of the experiment. Signals were recorded for 5 days prior to stimulation and throughout the 3-week stimulation period. The $10 \mathrm{~s}$ samples were used to create hourly averages that were further averaged over the $24 \mathrm{~h}$ period to generate daily means.

\section{Acute VNS}

Seven days after implantation, the vagus nerve was stimulated for $2 \mathrm{~h}$ (one burst of $20 \mathrm{~Hz}, 250 \mu$ s pulse width, $0.25 \mathrm{~mA}$ output current for $30 \mathrm{~s}$ every $5 \mathrm{~min}$ ) in six rats, whereas five rats served as sham controls. These stimulation parameters are very similar to those used clinically (Rush et al, 2005a, b). Thirty minutes after the $2 \mathrm{~h}$ vagus nerve or sham stimulation period, rats were anesthetized and perfused intracardially. All rats in this study were instrumented with radio telemetry transmitters.

\section{Chronic VNS Stimulation}

Rats $(n=9)$ received continuous VNS for 3 weeks with the same duty cycle and stimulus parameters used in the acute study. Nine rats served as sham controls. Six rats from the VNS group and six rats from the sham group received radio telemetry transmitters. Rats were perfused $30 \mathrm{~min}$ after the end of the experiment.

\section{Fos and $\Delta$ Fos $B$ Immunohistochemistry}

All rats were anesthetized with pentobarbital (50 mg/kg i.p.) and perfused with $0.1 \mathrm{M}$ phosphate-buffered saline (PBS) followed by $300-500 \mathrm{ml}$ of $4 \%$ paraformadlehyde in PBS. Brains were removed and placed in PBS with $30 \%$ sucrose for 3-4 days. Each brain was marked on the left side 
(ipsilateral to the VNS implant) and sectioned in a cryostat. Three serial sets of $40 \mu \mathrm{m}$ coronal sections from each brain were placed in cryoprotectant and stored at $-20^{\circ} \mathrm{C}$ until processed for immunohistochemistry (Cunningham et al, 2002).

Separate sets of serial sections were stained for either c-Fos (Rabbit anti-c-Fos Ab5, Calbiochem, San Diego, CA) or FosB (Goat anti-FosB (102; sc-46 g), Santa Cruz Biotechnology, Santa Cruz, CA) as previously described (Howe et al, 2004; Ji et al, 2005). The primary antibody used in this study does not discriminate between FosB and its splice variant $\Delta$ FosB. However, $\Delta$ FosB accumulates with chronic stimuli as a result of its long half-life, particularly the $37 \mathrm{kDa}$ isoform (Nestler, 2004), which is the isoform detected by the antibody used. For this reason, we refer to chronic stimulation increasing $\Delta$ FosB in this study although a contribution from FosB cannot be excluded. For c-Fos immunohistochemistry, sections were incubated with the primary antibody $(1: 30000)$ for $72 \mathrm{~h}$ at $4^{\circ} \mathrm{C}$. Sections processed for FosB staining were incubated with the primary antibody $(1: 5000)$ for $72 \mathrm{~h}$ at $4{ }^{\circ} \mathrm{C}$. The sections were incubated in biotyinlated horse anti-rabbit IgG or horse anti-goat IgG (Vector Laboratories, Berlingame, CA) diluted $1: 200$ in PBS for $2 \mathrm{~h}$ at room temperature. Sections were reacted with an avidin-peroxidase conjugate (Vectastain ABC Kit; Vector Laboratories) and PBS containing $0.04 \% 3,3^{\prime}$-diaminobenzidine hydrochloride and $0.04 \%$ nickel ammonium sulfate for 10 to $11 \mathrm{~min}$. Brain stem sections were then processed for dopamine- $\beta$-hydroxylase (DBH) immunofluorescence (Curtis et al, 1999; Grindstaff et al, 2000; Ji et al, 2005). Sections were mounted on gelatincoated slides, which were air-dried for 1-2 days, and coverslipped with Permount.

Forebrain regions included in the analysis were the paraventricular nucleus of the hypothalamus, supraoptic nucleus, amygdala, bed nucleus of the stria terminalis (BNST), paraventricular nucleus of the thalamus, vertical limb of the diagonal band of Broca, cingulate cortex, and insular cortex. The following brain stem regions were also analyzed: the dorsal raphe, LC, NTS, area postrema, caudal ventrolateral medulla, rostral ventrolateral medulla, and parabrachial nucleus. DBH immunofluorescence was used anatomically to define catecholamine-containing regions and ensure that sections were obtained from the same rostral-caudal plane for each set of sections from each rat. Tissue sections containing regions of interest were inspected using an Olympus microscope (IX 50) equipped for epifluorescence. Digital images were acquired using a Spot camera (SPOT RT Slider, Diagnostic Instruments, Sterling Heights, MI) connected to a Pentium computer running Spot Imaging software (v. 3.24). Some images were adjusted for brightness and contrast in order to standardize them for analysis. Regions of interest were identified using the rat brain stereotaxic atlas of Paxinos and Watson (1986). Three to six images were taken from each region for each animal bilaterally as previously described (Cunningham et al, 2002, 2007; Howe et al, 2004; Ji et al, 2005). The number of c-Fos or $\Delta$ FosB positive cells were recorded for each image and averaged for each animal. Counts were generated by observers who were blind to the treatment condition associated with the images (Cunningham et al, 2002, 2007; Howe et al, 2004; Ji et al, 2005).

\section{Forced Swim Test}

After 1 week of recovery, rats were trained and tested in the forced swim test (FST) using the method of Lucki (1997), with minor modifications. Training and test sessions were recorded by a video camera positioned above the swimming tank. On the training day, the rats were placed into a cylindrical tank $(60 \times 30 \mathrm{~cm})$ containing $25^{\circ} \mathrm{C}$ water at a depth of $35 \mathrm{~cm}$ for $15 \mathrm{~min}$. The first $5 \mathrm{~min}$ of the $15 \mathrm{~min}$ session were recorded on videotape. Water was changed between subjects. After $24 \mathrm{~h}$, the test session was carried out in the same manner for $5 \mathrm{~min}$, and recorded in its entirety.

One-half hour after the rats were removed from the water on the training day, they were injected either with saline or desipramine (DMI), or the vagal nerve stimulators were turned on (using the handheld computer). Rats received three sessions of VNS, using the stimulation parameters described above, for $2 \mathrm{~h}$ each, starting $30 \mathrm{~min}$ after the training session and then at 6.5 and $2.5 \mathrm{~h}$ before the test session. This sequence mimics the way drugs are usually given in the FST (Cryan et al, 2002a). Sham VNS controls were implanted with a 'dummy' simulator and electrodes placed around the left vagus nerve as described above. DMI, used at a dose of $15 \mathrm{mg} / \mathrm{kg}$, s.c., was injected $23.5,2.5$, and $0.5 \mathrm{~h}$ before the test session. A control group of rats received injections of $0.9 \% \mathrm{NaCl}$ at the same intervals. The videotaped behavior of the rats was scored using a timesampling technique to rate the predominant behavior over a $5 \mathrm{~s}$ interval as described by Lucki (1997). Immobility was defined as floating or no active movements made other than that necessary to keep the nose above the water. Swimming consisted of active motions throughout the swim tank and crossing into another quadrant, but not having the forepaws break the surface of the water. Climbing was defined as upward-directed movements of the forepaws against the wall and/or having the forepaws break or churn the surface of the water in vigorous swimming.

\section{Statistical Analysis}

Telemetry data from the acute and chronic studies were analyzed using separate two-way mixed effect analyses of variance (ANOVAs) and Newman-Keuls $t$-tests were used for post hoc analysis (SigmaStat, v. 2.03, Systat Software Inc., Point Richmond, CA). Data from the cell counts were analyzed by one-way ANOVA with Newman-Keuls $t$-test for post hoc analysis. Statistical analyses of the FST results were performed by Student's $t$-tests. All values are presented as mean \pm SEM. $P<0.05$ was considered statistically significant.

\section{RESULTS}

\section{Telemetry}

In the acute study, baseline values for rats receiving VNS were slightly higher for average MAP and somewhat lower for $\mathrm{HR}$ and RF compared with values for the sham group, but these differences were not statistically significant. Acute VNS did not significantly affect MAP, HR, RF, or activity as compared with the prestimulation baseline values or values for the sham group (Figure 1). Activity, as measured by the 

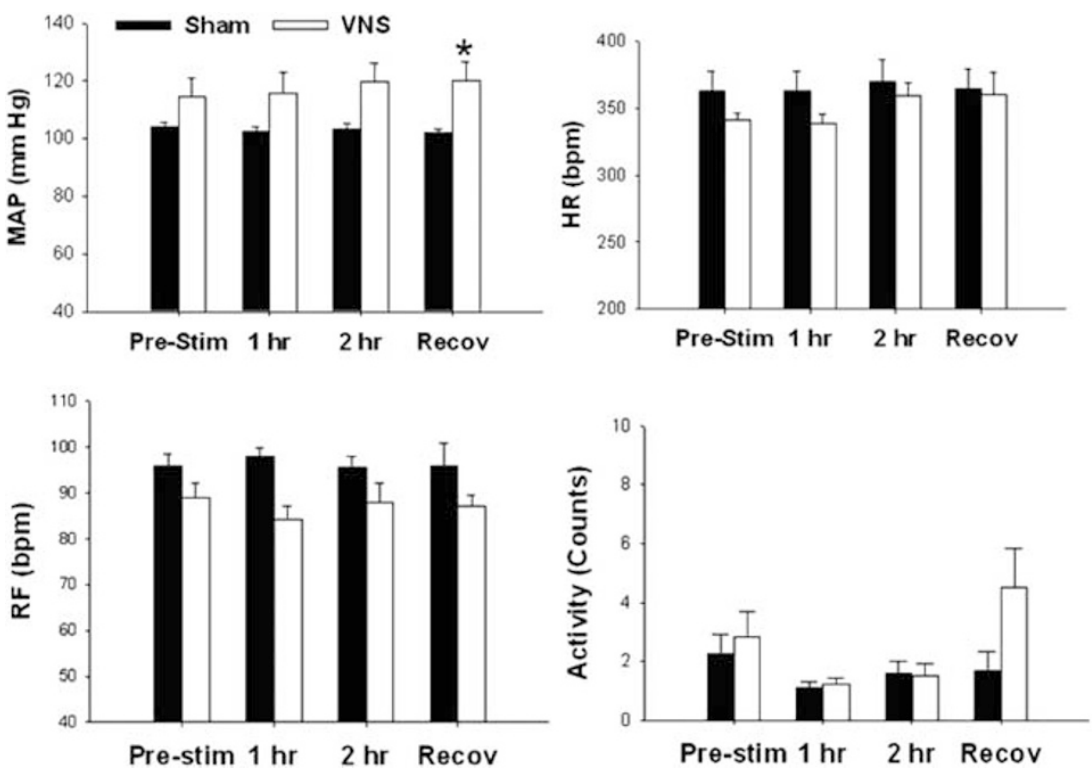

Figure I Effects of acute left vagus nerve stimulation (VNS) on mean arterial pressure (MAP), heart rate (HR), respiratory frequency (RF), and locomotor activity in rats receiving vagus nerve stimulation for $2 h(V N S, n=6)$ or sham controls $(n=5)$. Each variable was analyzed using separate two-way mixed effects ANOVA. *Significantly different from sham, $P<0.05$ (Student's Newman-Keuls t-test).

telemetry signal, was not significantly different between the two groups before or during VNS; there was a significant main effect for time due to an increase in activity during the recovery period (Figure 1).

Throughout the duration of the chronic study, the VNS group showed a trend toward a higher RF as compared to the sham group, but this trend was not statistically significant (Group $\mathrm{F}(1,9)=4.42, \quad P=0.065$; data not shown). HR decreased significantly during the stimulation period, but this decrease occurred in the sham rats as well as those that received VNS (Time $\mathrm{F}(25,225)=13.7$, $P<0.01)$. Activity in both groups also significantly varied across time during the experiment (Time $\mathrm{F}(25,225)=4.2$, $P<0.01)$. However, the differences were not statistically significant between the VNS and control groups either during the 5-day baseline period or during the 3 weeks of VNS for any of the measured variables. Thus, neither acute nor chronic VNS altered any of the parameters measured any differently than that seen in rats receiving sham VNS.

\section{Fos and $\Delta$ FosB Immunohistochemistry}

Several brain regions involved in reflex cardiovascular control, such as the caudal ventrolateral medulla, rostral ventrolateral medulla, and dorsal motor nucleus of the vagus were not affected by either acute or chronic VNS (Figure 2). These results are consistent with the telemetry data because no cardiovascular or respiratory effects were associated with either acute or chronic VNS. Other areas not affected significantly by either acute or chronic VNS, using these stimulation parameters, included the amygdala, supraoptic nucleus of the hypothalamus, diagonal band of Broca, and insular cortex.

In contrast, several regions of the CNS were affected by both acute and chronic VNS. The most commonly observed pattern of staining was best illustrated by the NTS. Acute
VNS significantly increased Fos staining bilaterally in the NTS with no effect on $\triangle$ FosB staining (Figures 2 and 3 ). After chronic VNS, both Fos and $\triangle$ FosB staining were increased bilaterally in the NTS (Figures 2 and 3). This pattern of staining was also observed in the LC and the ventral portion of the BNST as well as the parabrachial nucleus and the paraventricular nucleus of the hypothalamus (Figure 4).

Two regions showed a different pattern of staining associated with VNS. Both the DRN and the anterior cingulate cortex showed increased $\Delta$ FosB staining following chronic VNS, but Fos staining was not affected by either acute or chronic VNS (Figures 5 and 6). In the paraventricular nucleus of the thalamus, Fos staining was not affected by acute or chronic VNS. However, this region demonstrated high basal levels of $\Delta \mathrm{FosB}$ staining that significantly decreased in association with chronic VNS (Chronic Sham 47.3 \pm 4.6; Chronic VNS $25.5 \pm 2.8, P<0.01$, Newman-Keuls test). This was the only region in which a significant decrease in either Fos or $\triangle$ FosB staining occurred.

\section{Forced Swim Test}

As acute VNS elevated c-Fos in several brain areas, it was of interest to determine whether such stimulation caused a behavioral effect in the rats. The FST is a widely used behavioral test used to detect potential antidepressant-like activity (Cryan et al, 2005a; Porsolt et al, 1977). Consequently, we compared the effect of VNS in the FST with that produced by a standard antidepressant, DMI. As expected, DMI reduced immobility significantly in the FST and, also as expected (Cryan et al, 2005b), did so by an increase in climbing rather than swimming behavior (Figure 7). VNS also reduced immobility when administered similarly to DMI, (ie three times for $2 \mathrm{~h}$ each session between the 

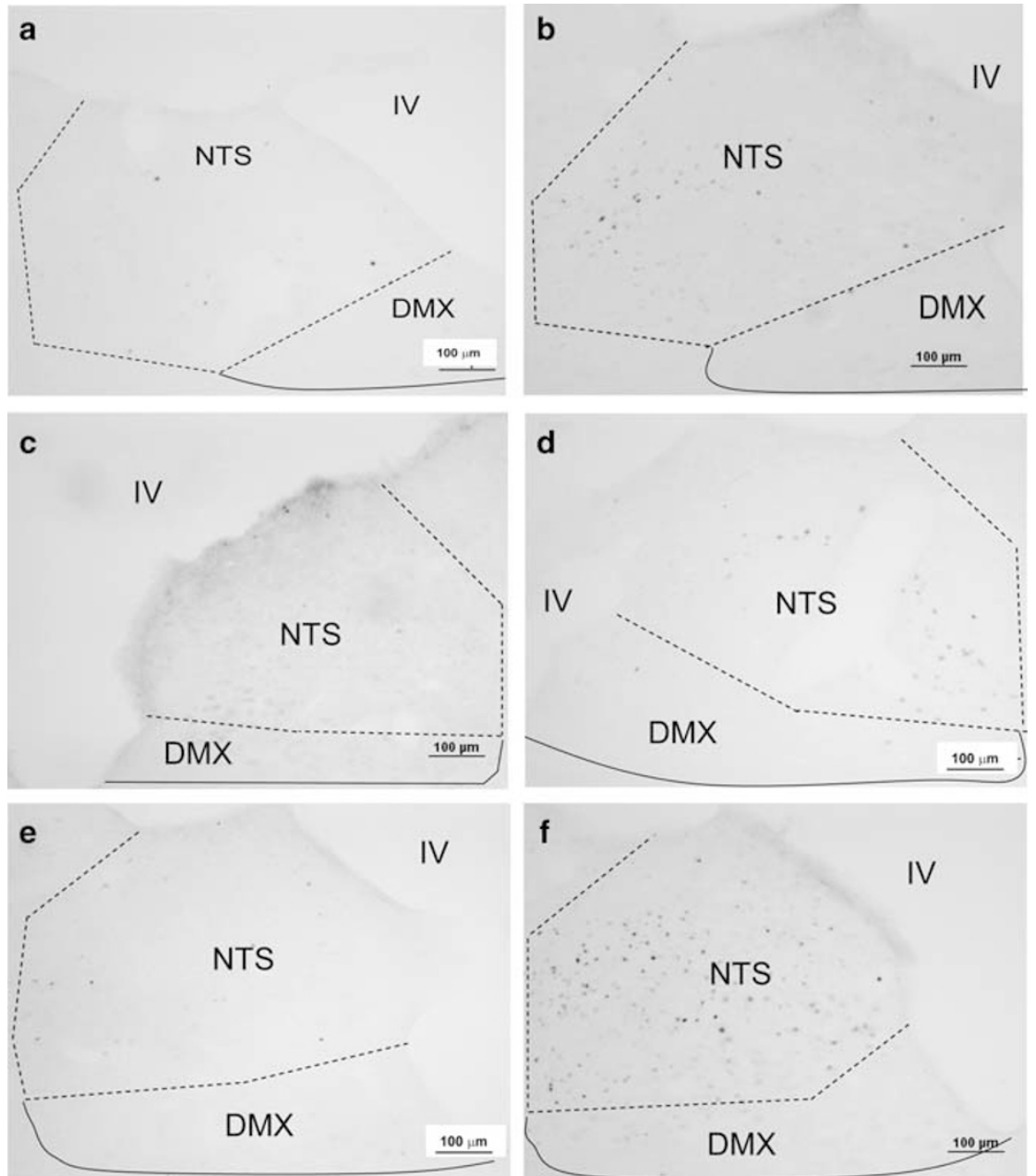

Figure 2 Representative digital images of c-Fos and $\Delta$ FosB staining in the nucleus of the solitary tract (NTS) and dorsal motor nucleus of the vagus (DMX) following either acute ( $2 \mathrm{~h}$ ) or chronic (3 weeks) VNS. (a) Fos staining for an acute sham animal. (b) Fos staining following acute VNS. (c) Fos staining from a chronic sham rat. (d) Fos staining after chronic VNS. (e) $\Delta$ FosB staining in a chronic sham rat. (f) $\Delta$ FosB staining after chronic VNS. Note that following VNS the nuclear staining that is characteristic of Fos and $\Delta$ FosB staining is located primarily in the NTS with little or no staining in the DMX
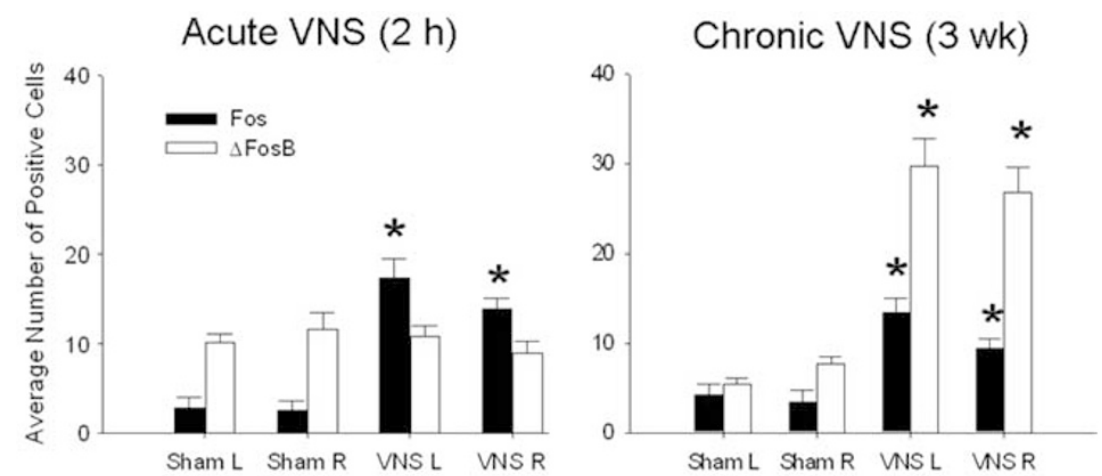

Figure 3 Summary data for the effects of acute (left) and chronic (right) vagus nerve stimulation (VNS) on Fos and $\Delta F o s B$ staining in the NTS. L and R on the $x$ axis indicate the left and right NTS from Sham and VNS-treated rats. In each group the electrode was implanted on the left vagus nerve. Acute VNS significantly increased Fos staining in both the left and right NTS without affecting $\Delta$ FosB staining. Chronic VNS was associated with significant bilateral increases in both Fos and $\Delta$ FosB. *Significantly different from sham, $P<0.05$ (Student's Newman-Keuls $t$-test).

training and test sessions). In contrast to DMI, though, the decrease in immobility resulted from an increase in swimming rather than from climbing behavior (Figure 7). During the training session, no significant differences in immobility were observed between the rats designated to receive either saline or DMI ( $33 \pm 2 v s 35 \pm 2$, respectively) or those that received sham VNS compared with those that received actual stimulation ( $23 \pm 2$ vs $21 \pm 2$, respectively). 


\section{Acute VNS (2h) \\ Acute VNS (2h) \\ Chronic VNS (3wk)}

\section{Locus Coeruleus}

Paraventricular Nucleus of the Hypothalamus
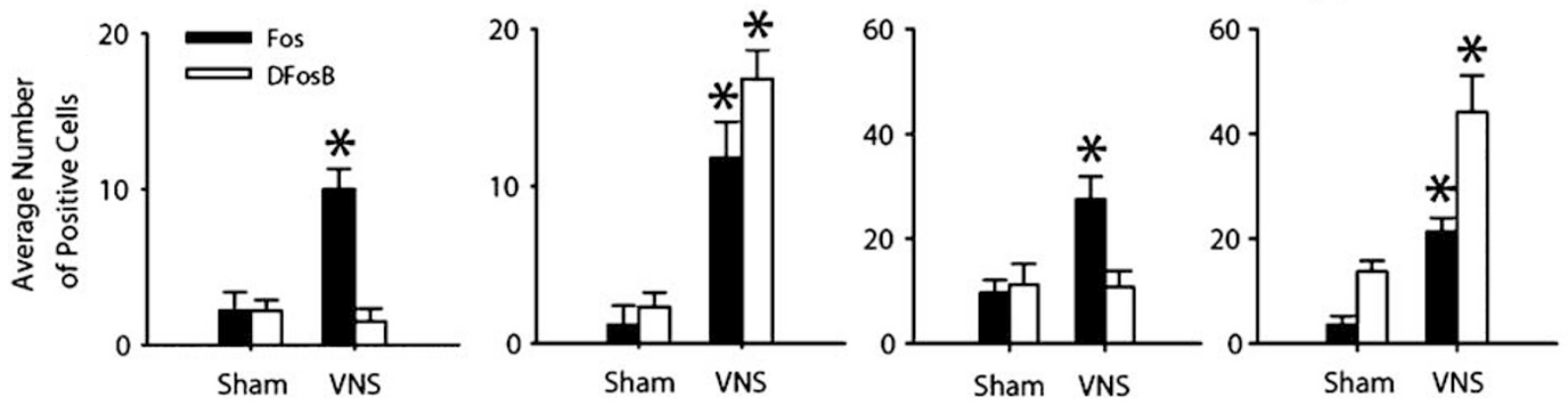

Bed Nucleus of the Stria Terminalis
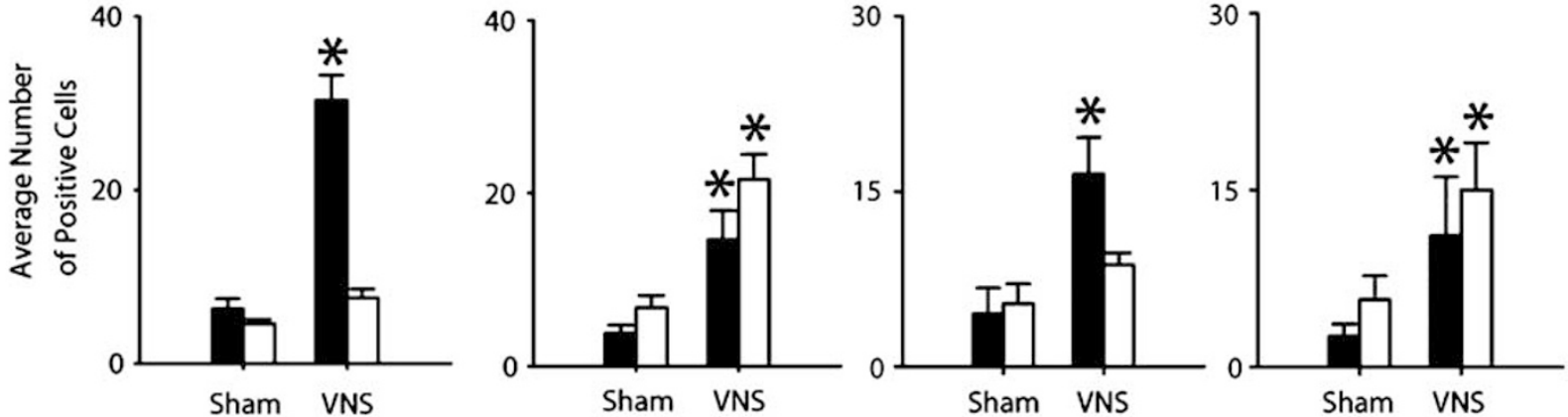

Figure 4 Effects of acute and chronic vagus nerve stimulation (VNS) on Fos and $\Delta$ FosB staining in the locus coeruleus, ventral bed nucleus of the stria terminalis, parabrachial nucleus, and paraventricular nucleus of the hypothalamus. Data from the ipsilateral side are presented in the graphs, although VNS produced significant increases bilaterally in both regions as was observed in the NTS. *Significantly different from sham, $P<0.05$ (Student's Newman-Keuls t-test).
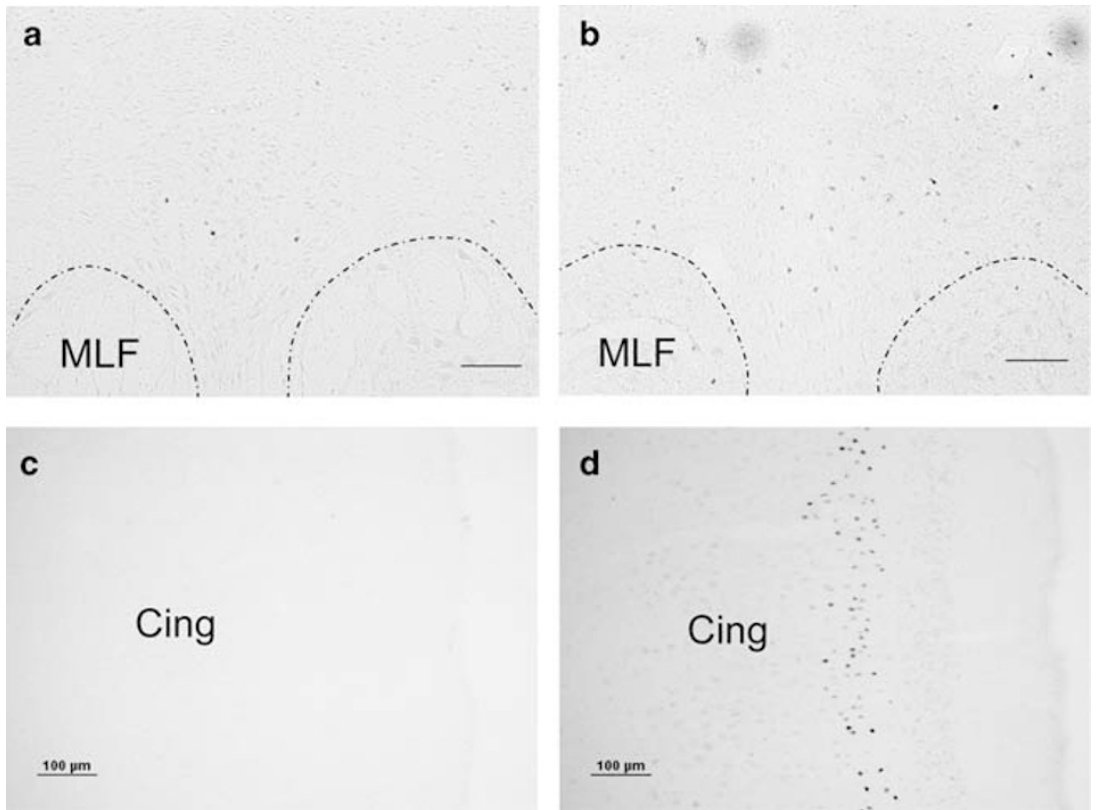

Figure 5 Representative digital images illustrating the effects of chronic VNS on $\Delta$ FosB staining in the dorsal raphe nucleus (DRN, top row) and anterior cingulate cortex (Cing, bottom row). (a and c) $\Delta$ FosB staining in a chronic sham rat. (b and d) $\Delta$ FosB staining following chronic VNS. MLF, medial longitudinal fasiculas.

Telemetry data from the acute VNS study indicated that VNS does not significantly affect general activity (Figure 1). Similarly, the telemetry data from the chronic VNS study also indicates that the first $6 \mathrm{~h}$ of VNS did not significantly affect general activity (6h prestimulation: Sham $2.2 \pm 0.6$, VNS $2.5 \pm 0.3 ; 6 \mathrm{~h}$ VNS: Sham $1.8 \pm 0.3$, VNS $2.2 \pm 0.3$; 


\section{Acute VNS (2 h) Chronic VNS (3 wk)}

\section{Dorsal Raphe Nucleus}
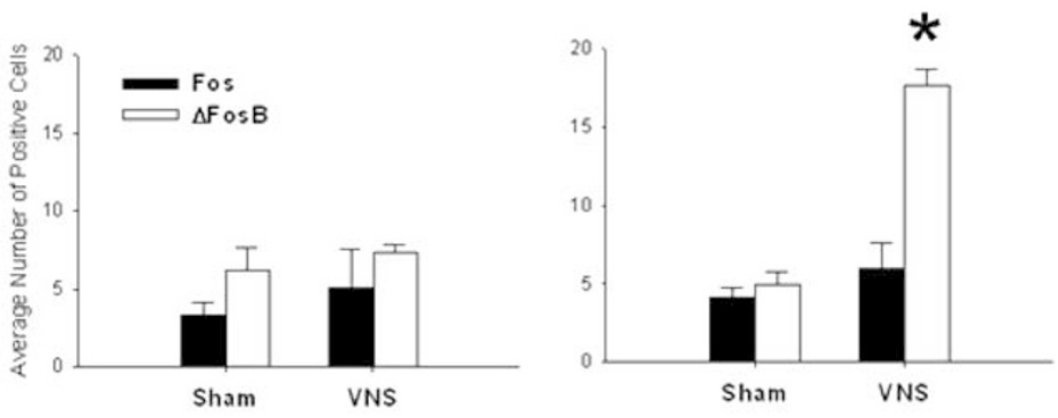

\section{Anterior Cingulate Cortex}
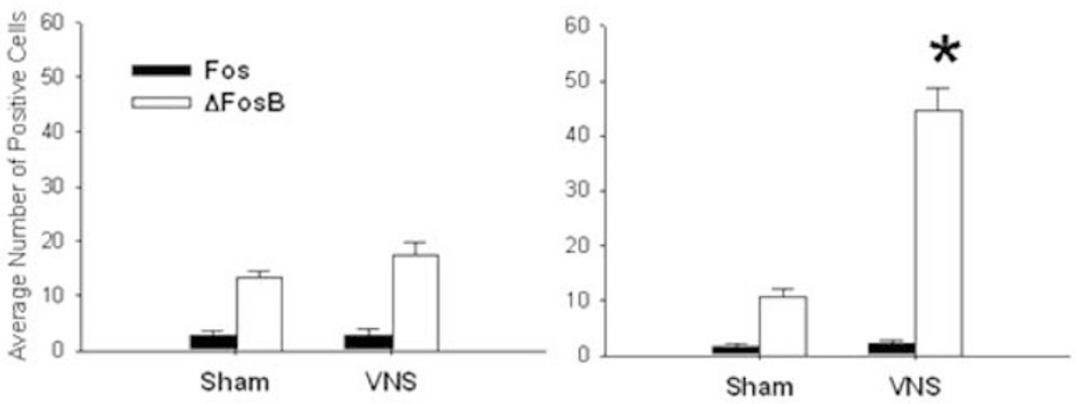

Figure 6 Effects of acute and chronic vagus nerve stimulation (VNS) on Fos and $\Delta$ FosB staining in the dorsal raphe nucleus (top) and the anterior cingulate cortex (bottom). In both regions, acute VNS was not associated with significant changes in either Fos or $\Delta$ FosB staining. Chronic VNS significantly increased $\Delta$ FosB staining with no effect on Fos. *Significantly different from sham, $P<0.05$ (Student's Newman-Keuls $t$-test).

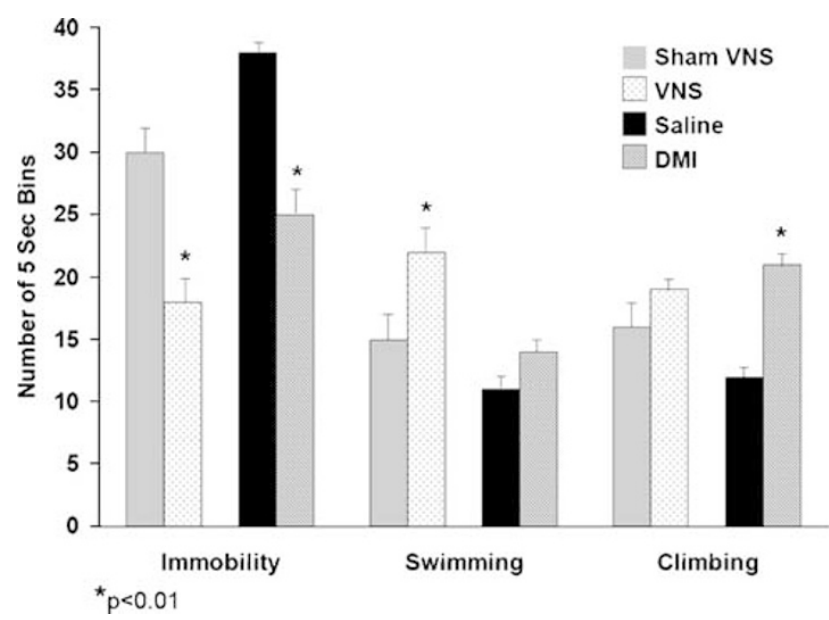

Figure 7 Effects of desipramine (DMI) treatment and vagus nerve stimulation (VNS) on immobility, swimming, and climbing activity in the forced swim test. *Significantly different from control value, $P<0.0$ I (Student's t-test).

$\mathrm{F}(1,10)=1.34, P>0.1)$. It is unlikely, therefore, that the decrease in immobility caused by VNS results from a general increase in locomotor activity.

\section{DISCUSSION}

Acute unilateral VNS in conscious rats, using clinically relevant stimulation parameters, increased staining for c-Fos bilaterally in the NTS and many, but not all, regions that receive its projections. Chronic VNS for 3 weeks also elevated c-Fos in the same areas as acute VNS. Whereas acute VNS never increased staining for $\triangle \mathrm{FosB}$, chronic VNS did so routinely. Even in the DRN and cingulate cortex where neither acute nor chronic VNS caused any increase in staining for c-Fos, staining for $\Delta$ FosB was increased significantly after chronic stimulation. Further, activation of brain using these stimulation parameters was sufficient to cause a behavioral effect, namely reduced immobility in the FST.

The electrode configuration used in this study was designed to create an anodal block at the distal end of the nerve (personal communication, William Buras, Cyberonics Inc.) so as to decrease the activation of vagal efferents. The anodal block is the result of current flowing from the anode to the cathode causing hyperpolarization at the anode and depolarization at the cathode. This hyperpolarization blocks the propagation of action potentials at the distal end of the electrode limiting the activation of vagal efferents. The anodal block seems to be effective with this stimulation 
because neither acute nor chronic stimulation caused changes in HR, MAP, or RF, even though central effects were produced by VNS. Consistent with this lack of peripheral autonomic effects was the absence of increased staining for either $c$-Fos or $\triangle \mathrm{FosB}$ in areas involved in cardiovascular or respiratory reflexes, such as the ventrolateral medulla or dorsal motor nucleus of the vagus (Dampney and Horiuchi, 2003; Guyenet, 2006). These findings support the likelihood that the VNS-induced increases centrally in either c-Fos or $\triangle$ FosB result directly from VNS and not indirectly from reflex activation of peripheral afferent inputs secondary to changes in MAP, $\mathrm{HR}$, or RF.

The absence of cardiovascular or respiratory effects using this electrode configuration and stimulation parameters is in contradistinction to previous studies on the effects of acute VNS on c-Fos in either the rabbit or the rat. In previously reported experiments, VNS produced marked effects on HR, MAP, and/or RF (Gieroba and Blessing, 1994; Naritoku et al, 1995; Osharina et al, 2006; Rutherfurd et al, 1992). Also, most of these studies were carried out using anesthetized animals and stimulation protocols with higher frequencies and duty cycles than the protocols used clinically and in the present study. Anesthesia also lowers activation of C fibers by VNS (Woodbury and Woodbury, 1990). Consequently, the effects reported in these earlier studies using anesthetized preparations may be distinct from those in unanesthetized animals. Such results, then, are unlikely to have much relevance for what occurs clinically when VNS is used in epilepsy or depression.

In the present study, stimulation of the vagus nerve for $2 \mathrm{~h}$ significantly increased staining for c-Fos in the NTS, parabrachial nucleus, LC, ventral BST, and paraventricular nucleus of the hypothalamus. Because the NTS is the primary termination site for vagal afferents (Kalia and Sullivan, 1982), the increase in staining for c-Fos is not unexpected. Also, the caudal two-thirds of the NTS is innervated by both the ipsilateral and the contralateral vagus nerves (Norgren, 1978; Ter Horst et al, 1989), so, bilateral activation of c-Fos in the NTS by stimulation of the left vagus was also anticipated. The NTS has a diverse network of afferent projections and it has been described as having three types or sets of afferent projections (Saper, 1995). First, the NTS has descending projections to autonomic preganglionic neurons. Second, it has projections to areas within the medullary reticular formation, such as the caudal and rostral ventrolateral medulla that are involved in cardiovascular and respiratory reflex control (Dampney, 1994; Guyenet, 2006). Third is a set of ascending projections from the NTS to midbrain, hypothalamic, and cortical regions that are involved in central autonomic control (Groves and Brown, 2005; Nemeroff et al, 2006). Included in this ascending system are direct projections to many regions, including cell bodies for noradrenergic or serotonergic neurons, ie the LC and DRN, respectively, with such neurons thought to be involved in the mechanisms of action of antidepressants (Lenox and Frazer, 2002). The LC has also been implicated in the anticonvulsant effects of VNS as well (Krahl et al, 1998). The NTS also sends indirect projections widely throughout the brain that originate from its direct projections (Castle et al, 2005; Groves and Brown,
2005; Nemeroff et al, 2006). For example, a recent study using transneuronal tract tracing demonstrated that visceral afferents from the NTS may influence the ventral hippocampus through a circuit that includes the LC, thalamus, and BST (Castle et al, 2005).

Human imaging studies of VNS also show widespread effects on subcortical and cortical regions (Barnes et al, 2003; Henry et al, 1998, 2004). Thus, stimulation of the vagus would be expected to cause widespread effects in many areas of brain including regions involved in affect and cognition. However, areas such as the amygdala, diagonal band of Broca, supraoptic nucleus, ventral hippocampus, DRN, and ventrolateral medulla did not show increases in cFos staining even though they also receive either direct projections from the NTS and/or innervation from areas to which the NTS projects (Castle et al, 2005; Dampney, 1994; Nemeroff et al, 2006). At least as measured by c-Fos, the stimulation parameters used in this study are not causing sufficient stimulation of the NTS to result in increased c-Fos staining in all of its projection areas. It is also possible that in some of these regions, such as the amygdala or supraoptic nucleus, VNS may be associated with inhibitory effects that would not be reflected by changes in c-Fos staining. Negative results must be interpreted with caution because previous studies have demonstrated that some populations of neurons require sustained stimulation in order to produce c-Fos (Dampney and Horiuchi, 2003; Dragunow and Faull, 1989).

One previous study measured c-Fos in rats after acute VNS using the Cyberonics stimulator (Naritoku et al, 1995). Anesthetized rats received VNS for $3 \mathrm{~h}$ using the same $10 \%$ duty cycle that we used although the frequency $(30 \mathrm{~Hz})$, current $(1 \mathrm{~mA})$, and stimulus pulse duration $(0.5 \mathrm{~ms})$ were greater. Cardiovascular or respiratory parameters were not measured. In contrast to our results, Naritoku et al (1995) found high staining for c-Fos in a nucleus of the amygdala and cingulate cortex as well as the dorsal motor nucleus of the vagus and the NTS. Similar to our results, they found activation in the LC but not in the DRN. Given the difference in stimulation parameters and the use of anesthetized rats, some differences in results are to be expected.

Of course, VNS is administered chronically when used clinically. Consequently, it was of interest to compare the pattern of activation in areas of brain after acute $(2 \mathrm{~h})$ or chronic (3 weeks) VNS. $\triangle$ FosB was measured to reflect chronic activation (Cunningham et al, 2007; Gottlieb et al, 2006; Herdegen and Leah, 1998; Ji et al, 2005; Nestler, 2004). There are four major protein members of the Fos family: c-Fos, FosB, Fra-1, and Fra-2 (Herdegen and Leah, 1998; Kovacs, 1998; Nestler, 2004). In general, maximal levels of c-Fos protein occur within $1-3 \mathrm{~h}$ of stimulus exposure and then disappear in 4-6 h, whereas FosB and its splice variant, $\Delta$ FosB, show a more delayed activation but persist longer (Chen et al, 1997). The accumulation of $\Delta$ FosB with chronic stimuli is a result of its long half-life, particularly the $37 \mathrm{kDa}$ isoform (Nestler, 2004), which is the isoform detected along with FosB by the antibody used in this study.

In all areas in which acute VNS increased staining for c-Fos, chronic VNS did as well, although the increases were generally somewhat less than those seen with acute stimulation. As anticipated, chronic VNS elevated $\Delta$ FosB 
and it did so in all areas where either acute or chronic VNS increased staining for $\mathrm{c}$-Fos. Increased $\triangle \mathrm{FosB}$ staining associated with chronic VNS is likely due to the accumulation of this protein resulting from intermittent activation in these regions over the 3 weeks of stimulation. Increased c-Fos staining after chronic VNS also could be related to the intermittent nature of the stimulation protocol (ie rats only receive stimulation for $10 \%$ of the time). However, increased c-Fos staining associated with chronic experimental manipulations such as water deprivation and hypertension has been previously reported (Cunningham et al, 2007; Ji et al, 2005; Miyata et al, 2001).

Chronic VNS also increased $\triangle \mathrm{FosB}$ in the DRN and the cingulate cortex where increases in c-Fos staining had not been seen with either acute or chronic stimulation. These results indicate that VNS at these stimulation parameters produces acute activation of the LC but only a more delayed activation of the DRN and a cortical area, the cingulate. The activation of the cingulate by chronic VNS contrasts with the results of imaging studies in patients in which decreased subgenual cingulate (Cg25) activity was found in association with clinical response to various types of antidepressant treatments (Goldapple et al, 2004; Mayberg et al, 2000; Nobler et al, 2001). Also chronic deep brain stimulation of the white matter tracts adjacent to the subgenual cingulate produced a marked beneficial effect in four of six patients with treatment refractory depression (Mayberg et al, 2005). The cingulate cortex would appear to be an important area to study in future research on antidepressant treatments.

The temporal pattern of activation of the LC and DRN is of interest given the importance of norepinephrine and serotonin in the mechanism of action of antidepressants (Lenox and Frazer, 2002) and the continuing improvement in depression produced over time by VNS (Groves et al, 2005; Rush et al, 2005b). The time course of activation in the present study is consistent with the results of Dorr and Debonnel (2006) who measured the ability of VNS to increase the firing rate of LC or DRN neurons. These investigators also used stimulators supplied by Cyberonics Inc. and the same stimulation parameters used by us. In conscious rats, stimulation of the vagus for as little as $1 \mathrm{~h}$ produced increases in the firing rate of LC neurons, with the firing rate further increasing linearly for up to 90 days. By contrast, no increases in the firing rate of neurons in the DRN were seen for up to 3 days of stimulation; however, their firing rate increased significantly after 14 days of VNS with the firing rates increasing even further after 90 days of stimulation. Groves et al (2005) also reported recently that acute stimulation of the vagus nerve in anesthetized rats increased the firing rate of LC neurons. Acute activation of the LC by VNS is consistent with reports that the LC seems to be necessary for its acute antiseizure activity in an animal model (Krahl et al, 1998) and that acute VNS increases extracellular norepinephrine (Roosevelt et al, 2006).

In view of our results with VNS, of relevance are studies that have measured mRNA for $c$-fos or c-Fos protein after antidepressant treatments. Essentially all studies were conducted after acute ( $30 \mathrm{~min}-2 \mathrm{~h}$ ) administration of antidepressants. A reasonably consistent observation is that administration of pharmacologically diverse antidepressants (SSRIs, selective NRIs, MAOIs, mirtazapine) increase $c$-fos or c-Fos in the central nucleus of the amygdala (CeA) and BNST (Beck, 1995; Duncan et al, 1996; Fraga et al, 2005; Miyata et al, 2005; Morelli et al, 1999; Slattery et al, 2005; Sumner et al, 2004; Thomsen and Helboe, 2003). This increase was also seen in female rats treated acutely with fluoxetine (Torres et al, 1998). Most studies report no increase in the DRN, although Fraga et al (2005) did find an increase after administration of fluoxetine. When increases in the LC have been reported, it has been upon administration of the SSRI fluoxetine (Fraga et al, 2005; Slattery et al, 2005; Sumner et al, 2004) but, with one exception (Beck, 1995), not with other types of antidepressants. In general, increases have not been reported in the hippocampus and many other regions of brain and effects in cortical areas have been quite inconsistent. Acute electroconvulsive shock (ECS) also increases c-Fos in cortex and hippocampus (Cole et al, 1990; Hope et al, 1994; Morinobu et al, 1997; Winston et al, 1990). Interestingly, chronic treatment of rats with either paroxetine (Muigg et al, 2007) or citalopram (Kuipers et al, 2006) does not increase c-Fos in any brain region examined, including the $\mathrm{CeA}$. We have found only one study of chronic antidepressant treatment on $\triangle F$ FosB. In that study, ECS increased $\Delta$ FosB in cortex and hippocampus after 2 days and the effect became maximal after 4 days (Hope et al, 1994).

Not surprisingly, comparing such results with the acute VNS results in the present study reveals both similarities and differences. Perhaps most striking is that VNS did not increase c-Fos in any amygdaloid nuclei, although it did in the BNST. Also, VNS increased c-Fos in the LC, whereas this was either not seen or seen inconsistently with antidepressants other than fluoxetine. Similar to the result with antidepressants, acute VNS did not increase c-Fos in the DRN. Also, we found chronic VNS to increase c-Fos (as well as $\Delta$ FosB) in areas of brain, whereas this has not yet been observed with chronic administration of SSRIs. In view of these effects of VNS, it would be useful to investigate whether antidepressants produced effects in areas not examined previously, such as the NTS or parabrachial nucleus. Whether such differences in effects between VNS and antidepressants contributes to the utility of VNS in treatment-resistant depression remains to be determined.

Given the limited activation of brain caused by these stimulation parameters, it was of interest to see if they would produce a behavioral effect. To explore this, the FST was used. This test was originally described by Porsolt et al (1977) to detect antidepressant-like activity in rats and has become one of the more widely, if not the most widely, used procedure for detecting antidepressant-like activity in rodents (Cryan et al, 2005a,b). The antidepressants are often given three times between the first and second exposure to the swim tank. Consistent with many other reports (Cryan et al, 2002b), when DMI, the noradrenergic reuptake inhibitor, was administered in this way it caused a decrease in immobility that was due to an increase in climbing, but not swimming, behavior. There are substantial data that antidepressants that act selectively through norepinephrine increase climbing but not swimming behavior (see Cryan et al, 2002b), whereas serotonergic antidepressants reduce immobility by increasing swimming 
activity rather than climbing activity (Dziedzicka-Wasylewska et al, 2006; Page et al, 1999; Xu et al, 2000). VNS, when administered for thee sessions of $2 \mathrm{~h}$ each between the two swim sessions, produced a comparable reduction in immobility to DMI. These results are consistent with those of Krahl et al (2004) who found that 30 min of continuous VNS each day for 4 days reduced immobility in the FST. Thus, stimulation with these clinically relevant parameters can produce an antidepressant-like effect in rats. However, the decrease in immobility caused by VNS was due to an increase in swimming rather than in climbing activity. Given this difference, it is reasonable to speculate that the effect of VNS in the FST is more likely due to a serotonergic rather than to a noradrenergic mechanism. However, the results with c-Fos show no effect of VNS in the DRN after $2 \mathrm{~h}$ of stimulation even though an effect was found in the LC. In the forced swim experiment, VNS was given three times for $2 \mathrm{~h}$ each session over $23 \mathrm{~h}$. Future experiments should explore whether such a paradigm causes an increase in either c-Fos or $\Delta$ FosB in the raphe and whether lesions of serotonergic or noradrenergic neurons influence the effects of VNS in the FST.

In conclusion, the present study identified potential sites in brain where VNS may produce its clinically significant effects. A limited number of sites showed activation, as measured by c-Fos, after acute stimulation with chronic stimulation showing activation of the same sites as acute stimulation plus two more, namely the DRN and the anterior cingulate cortex. Future research will be necessary to determine the functional implications of activation of these sites.

\section{ACKNOWLEDGEMENTS}

We acknowledge the technical assistance of M HerreraRosales and MA Martinez and thank S Westerman for her assistance in preparing the manuscript. This work was partially funded by a grant from Cyberonics Inc.

\section{DISCLOSURE/CONFLICT OF INTEREST}

The authors declare that this research was funded in part by an extramural research grant from Cyberonics Inc. the manufacturer of the VNS device used in this study.

The authors declare that over the past 3 years JTC, SWM, and AF have received fiscal compensation as consultants for Cyberonics Inc. In addition, AF has served as a consultant or on advisory boards for Lilly, Lundbeck, Sepracor, and Wyeth. In addition, GG declares that except for income received from my primary employer, no financial support or compensation has been received from any individual or corporate entity over the past 3 years for research or professional service, and there are no personal financial holdings that could be perceived as constituting a potential conflict of interest.

\section{REFERENCES}

Ansah T, Wage L, Shockley D (1996). Changes in locomotor activity, core temperature, and heart rate in response to repeated cocaine administration. Physiol Behav 59: 1261-1267.
Barnes A, Duncan R, Chisholm JA, Lindsay K, Patterson J, Wyper D (2003). Investigation into the mechanisms of vagus nerve stimulation for the treatment of intractable epilepsy, using ${ }^{99 m}$ Tc-HMPAO SPET brain images. Eur J Nucl Med Mol Imaging 30: 301-305.

Beck CH (1995). Acute treatment with antidepressant drugs selectively increases the expression of $\mathrm{c}$-fos in the rat brain. J Psychiatry Neurosci 20: 25-32.

Castle M, Comoli E, Loewy AD (2005). Autonomic brainstem nuclei are linked to the hippocampus. Neuroscience 134: 657-669.

Chen J, Kelz MB, Hope BT, Nakabeppu Y, Nestler EJ (1997). Chronic Fos-related antigens: stable variants of deltaFosB induced in brain by chronic treatments. J Neurosci 17: 4933-4941.

Cole AJ, Abu-Shakra S, Saffen DW, Baraban JM, Worley PF (1990). Rapid rise in transcription factor mRNAs in rat brain after electroshock-induced seizures. J Neurochem 55: 1920-1927.

Cryan JF, Markou A, Lucki I (2002a). Assessing antidepressant activity in rodents: recent developments and future needs. Trends Pharmacol Sci 23: 238-245.

Cryan JF, Page ME, Lucki I (2002b). Noradrenergic lesions differentially alter the antidepressant-like effects of reboxetine in a modified forced swim test. Eur J Pharmacol 436: 197-205.

Cryan JF, Page ME, Lucki I (2005b). Differential behavioral effects of the antidepressants reboxetine, fluoxetine, and moclobemide in a modified forced swim test following chronic treatment. Psychopharmacology (Berl) 182: 335-344.

Cryan JF, Valentino RJ, Lucki I (2005a). Assessing substrates underlying the behavioral effects of antidepressants using the modified rat forced swimming test. Neurosci Biobehav Rev 29: 547-569.

Cunningham JT, Grindstaff RJ, Grindstaff RR, Sullivan MJ (2002). Fos immunoreactivity in the diagonal band and the perinuclear zone of the supraoptic nucleus after hypertension and hypervolaemia in unanaesthetized rats. J Neuroendocrinol 14: 219-227.

Cunningham JT, Herrera-Rosales M, Martinez MA, Mifflin S (2007). Identification of active central nervous system sites in renal wrap hypertensive rats. Hypertension 49: 653-658.

Curran T, Morgan JI (1995). Fos: an immediate-early transcription factor in neurons. J Neurobiol 26: 403-412.

Curtis KS, Cunningham JT, Heesch CM (1999). Fos expression in brain stem nuclei of pregnant rats after hydralazine-induced hypotension. Am J Physiol 277(2 Part 2): R532-R540.

Dampney RA (1994). Functional organization of central pathways regulating the cardiovascular system. Physiol Rev 74: 323-364.

Dampney RA, Horiuchi J (2003). Functional organisation of central cardiovascular pathways: studies using c-fos gene expression. Prog Neurobiol 71: 359-384.

Dorr AE, Debonnel G (2006). Effect of vagus nerve stimulation on serotonergic and noradrenergic transmission. J Pharmacol Exp Ther 318: 890-898.

Dragunow M, Faull R (1989). The use of c-fos as a metabolic marker in neuronal pathway tracing. J Neurosci Methods 29: 261-265.

Duncan GE, Knapp DJ, Johnson KB, Breese GR (1996). Functional classification of antidepressants based on antagonism of swim stress-induced fos-like immunoreactivity. J Pharmacol Exp Ther 277: 1076-1089.

Dziedzicka-Wasylewska M, Faron-Gorecka A, Kusmider M, Drozdowska E, Rogoz Z, Siwanowicz J et al (2006). Effect of antidepressant drugs in mice lacking the norepinephrine transporter. Neuropsychopharmacology 31: 2424-2432.

Foley JO, DuBois F (1937). Quantitative studies of the vagus nerve in the cat. I. The ratio of sensory motor studies. J Comp Neurol 67: 49-67.

Fraga IC, Fregoneze JB, Carvalho FL, Dantas KB, Azevedo CS, Pinho $\mathrm{CB}$ et al (2005). Acute fluoxetine administration 
differentially affects brain C-Fos expression in fasted and refed rats. Neuroscience 134: 327-334.

Gieroba ZJ, Blessing WW (1994). Fos-containing neurons in medulla and pons after unilateral stimulation of the afferent abdominal vagus in conscious rabbits. Neuroscience 59: 851-858.

Goldapple K, Segal Z, Garson C, Lau M, Bieling P, Kennedy S et al (2004). Modulation of cortical-limbic pathways in major depression: treatment-specific effects of cognitive behavior therapy. Arch Gen Psychiatry 61: 34-41.

Gottlieb HB, Ji LL, Jones H, Penny ML, Fleming T, Cunningham JT (2006). Differential effects of water and saline intake on water deprivation-induced c-Fos staining in the rat. Am J Physiol Regul Integr Comp Physiol 290: R1251-R1261.

Grindstaff RJ, Grindstaff RR, Sullivan MJ, Cunningham JT (2000). Role of the locus ceruleus in baroreceptor regulation of supraoptic vasopressin neurons in the rat. Am J Physiol Regul Integr Comp Physiol 279: R306-R319.

Groves DA, Bowman EM, Brown VJ (2005). Recordings from the rat locus coeruleus during acute vagal nerve stimulation in the anaesthetised rat. Neurosci Lett 379: 174-179.

Groves DA, Brown VJ (2005). Vagal nerve stimulation: a review of its applications and potential mechanisms that mediate its clinical effects. Neurosci Biobehav Rev 29: 493-500.

Guyenet PG (2006). The sympathetic control of blood pressure. Nat Rev Neurosci 7: 335-346.

Henry TR, Bakay RA, Pennell PB, Epstein CM, Votaw JR (2004). Brain blood-flow alterations induced by therapeutic vagus nerve stimulation in partial epilepsy: II. prolonged effects at high and low levels of stimulation. Epilepsia 45: 1064-1070.

Henry TR, Bakay RA, Votaw JR, Pennell PB, Epstein CM, Faber TL et al (1998). Brain blood flow alterations induced by therapeutic vagus nerve stimulation in partial epilepsy: I. Acute effects at high and low levels of stimulation. Epilepsia 39: 983-990.

Herdegen T, Leah JD (1998). Inducible and constitutive transcription factors in the mammalian nervous system: control of gene expression by Jun, Fos and Krox, and CREB/ATF proteins. Brain Res Brain Res Rev 28: 370-490.

Hope BT, Kelz MB, Duman RS, Nestler EJ (1994). Chronic electroconvulsive seizure (ECS) treatment results in expression of a long-lasting AP-1 complex in brain with altered composition and characteristics. J Neurosci 14: 4318-4328.

Howarth F, Jacobson M, Naseer O, Adeghate E (2005). Short- term effects of streptozotocin- induced diabetes on the electrocardiogram, physical activity and body temperature in rats. Exp Physiol 90: 229-237.

Howe BM, Bruno SB, Higgs KA, Stigers RL, Cunningham JT (2004). FosB expression in the central nervous system following isotonic volume expansion in unanesthetized rats. Exp Neurol 187: 190-198.

Ji LL, Fleming T, Penny ML, Toney GM, Cunningham JT (2005). Effects of water deprivation and rehydration on c-Fos and FosB staining in the rat supraoptic nucleus and lamina terminalis region. Am J Physiol Regul Integr Comp Physiol 288: R311-R321.

Kalia M, Sullivan JM (1982). Brainstem projections of sensory and motor components of the vagus nerve in the rat. J Comp Neurol 211: 248-265.

Kawashima K, Amano H, Fujimoto K, Suzuki T, Fujii T, Mochizuki $S$ et al (1996). Effect of repeated administration of KT3-671, a nonpeptide AT1 receptor antagonist, on diurnal variation in blood pressure, heart rate, and locomotor acitivity in strokeprone spontanesouly hypertensive rats as determined by radiotelemetry. J Cardiovasc Pharmacol 27: 411-416.

Kovacs KJ (1998). c-Fos as a transcription factor: a stressful (re)view from a functional map. Neurochem Int 33: 287-297.

Krahl SE, Clark KB, Smith DC, Browning RA (1998). Locus coeruleus lesions suppress the seizure-attenuating effects of vagus nerve stimulation. Epilepsia 39: 709-714.
Krahl SE, Senanayake SS, Pekary AE, Sattin A (2004). Vagus nerve stimulation (VNS) is effective in a rat model of antidepressant action. J Psychiatr Res 38: 237-240.

Kuipers SD, Trentani A, Westenbroek C, Bramham CR, Korf J, Kema IP et al (2006). Unique patterns of FOS, phospho-CREB and BrdU immunoreactivity in the female rat brain following chronic stress and citalopram treatment. Neuropharmacology 50: 428-440.

Lenox RH, Frazer A (2002). Mechanism of action of antidepressants and mood-stabilizers. In: Davis KL, Charney D, Coyle JT, Nemeroff C (eds). Neuropsychopharmacology, The Fifth Generation of Progress. Lippincott Williams \& Wilkins: Philadelphia. pp 1139-1163.

Lucki I (1997). The forced swimming test as a model for core and component behavioral effects of antidepressant drugs. Behav Pharmacol 8: 523-532.

Mayberg HS, Brannan SK, Tekell JL, Silva JA, Mahurin RK, McGinnis S et al (2000). Regional metabolic effects of fluoxetine in major depression: serial changes and relationship to clinical response. Biol Psychiatry 48: 830-843.

Mayberg HS, Lozano AM, Voon V, McNeely HE, Seminowicz D, Hamani $C$ et al (2005). Deep brain stimulation for treatmentresistant depression. Neuron 45: 651-660.

Meerlo P, Sgoifo A, Boer S, Koolhaas J (1999). Long-lasting consequences of a social conflict in rats: behavior during the interaction predicts subsequent changes in daily rhythms of heart rate, temperature, and activity. Behav Neurosci 113: 1283-1290.

Miyata S, Hamamura T, Lee Y, Miki M, Habara T, Oka T et al (2005). Contrasting Fos expression induced by acute reboxetine and fluoxetine in the rat forebrain: neuroanatomical substrates for the antidepressant effect. Psychopharmacology (Berl) 177: 289-295.

Miyata S, Tsujioka $\mathrm{H}$, Itoh $\mathrm{M}$, Matsunaga $\mathrm{W}$, Kuramoto $\mathrm{H}$, Kiyohara T (2001). Time course of Fos and Fras expression in the hypothalamic supraoptic neurons during chronic osmotic stimulation. Brain Res Mol Brain Res 90: 39-47.

Morelli M, Pinna A, Ruiu S, Del Zompo M (1999). Induction of Fos-like-immunoreactivity in the central extended amygdala by antidepressant drugs. Synapse 31: 1-4.

Morinobu S, Strausbaugh H, Terwilliger R, Duman RS (1997). Regulation of c-Fos and NGF1-A by antidepressant treatments. Synapse 25: 313-320.

Muigg P, Hoelzl U, Palfrader K, Neumann I, Wigger A, Landgraf R et al (2007). Altered brain activation pattern associated with drug-induced attenuation of enhanced depression-like behavior in rats bred for high anxiety. Biol Psychiatry 61: 782-796.

Naritoku DK, Terry WJ, Helfert RH (1995). Regional induction of fos immunoreactivity in the brain by anticonvulsant stimulation of the vagus nerve. Epilepsy Res 22: 53-62.

Nemeroff CB, Mayberg HS, Krahl SE, McNamara J, Frazer A, Henry TR et al (2006). VNS therapy in treatment-resistant depression: clinical evidence and putative neurobiological mechanisms. Neuropsychopharmacology 31: 1345-1355.

Nestler EJ (2004). Molecular mechanisms of drug addiction. Neuropharmacology 47(Suppl 1): 24-32.

Nobler MS, Oquendo MA, Kegeles LS, Malone KM, Campbell CC, Sackeim HA et al (2001). Decreased regional brain metabolism after ect. Am J Psychiatry 158: 305-308.

Norgren R (1978). Projections from the nucleus of the solitary tract in the rat. Neuroscience 3: 207-218.

Osharina V, Bagaev V, Wallois F, Larnicol N (2006). Autonomic response and Fos expression in the NTS following intermittent vagal stimulation: Importance of pulse frequency. Auton Neurosci: Basic Clin 126-127: 72-80.

Page ME, Detke MJ, Dalvi A, Kirby LG, Lucki I (1999). Serotonergic mediation of the effects of fluoxetine, but not desipramine, in 
the rat forced swimming test. Psychopharmacology (Berl) 147: 162-167.

Paxinos G, Watson C (1986). The Rat Brain in Stereotaxic Coordinates. Academic Press: New York.

Porsolt RD, Le Pichon M, Jalfre M (1977). Depression: a new animal model sensitive to antidepressant treatments. Nature 266: 730-732.

Roosevelt RW, Smith DC, Clough RW, Jensen RA, Browning RA (2006). Increased extracellular concentrations of norepinephrine in cortex and hippocampus following vagus nerve stimulation in the rat. Brain Res 1119: 124-132.

Rush AJ, Marangell LB, Sackeim HA, George MS, Brannan SK, Davis SM et al (2005a). Vagus nerve stimulation for treatmentresistant depression: a randomized, controlled acute phase trial. Biol Psychiatry 58: 347-354.

Rush AJ, Sackeim HA, Marangell LB, George MS, Brannan SK, Davis SM et al (2005b). Effects of 12 months of vagus nerve stimulation in treatment-resistant depression: a naturalistic study. Biol Psychiatry 58: 355-363.

Rutherfurd SD, Widdop RE, Sannajust F, Louis WJ, Gundlach AL (1992). Expression of c-fos and NGFI-A messenger RNA in the medulla oblongata of the anaesthetized rat following stimulation of vagal and cardiovascular afferents. Brain Res Mol Brain Res 13: $301-312$.

Sackeim HA, Brannan SK, John Rush A, George MS, Marangell LB, Allen J (2007). Durability of antidepressant response to vagus nerve stimulation (VNSTM). Int $J$ Neuropsychopharmacol (originally published online by Cambridge University Press, 9 February 2007, http://journals.cambridge.org/ 193378).

Saper CB (1995). Central autonomic system. In: Paxinos G (ed). The Rat Nervous System. Academic Press: San Diego, CA. pp 107-135.

Senba E, Ueyama T (1997). Stress-induced expression of immediate early genes in the brain and peripheral organs of the rat. Neurosci Res 29: 183-207.

Slattery DA, Morrow JA, Hudson AL, Hill DR, Nutt DJ, Henry B (2005). Comparison of alterations in c-fos and Egr-1 (zif268) expression throughout the rat brain following acute adminis- tration of different classes of antidepressant compounds. Neuropsychopharmacology 30: 1278-1287.

Sumner BE, Cruise LA, Slattery DA, Hill DR, Shahid M, Henry B (2004). Testing the validity of c-fos expression profiling to aid the therapeutic classification of psychoactive drugs. Psychopharmacology (Berl) 171: 306-321.

Takayama K, Suzuki T, Miura M (1994). The comparison of effects of various anesthetics on expression of Fos protein in the rat brain. Neurosci Lett 176: 59-62.

Ter Horst GJ, de Boer P, Luiten PG, van Willigen JD (1989). Ascending projections from the solitary tract nucleus to the hypothalamus. A Phaseolus vulgaris lectin tracing study in the rat. Neuroscience 31: 785-797.

Thomsen C, Helboe L (2003). Regional pattern of binding and c-Fos induction by (R)- and (S)-citalopram in rat brain. Neuroreport 14: 2411-2414.

Torres G, Horowitz JM, Laflamme N, Rivest S (1998). Fluoxetine induces the transcription of genes encoding c-fos, corticotropinreleasing factor and its type 1 receptor in rat brain. Neuroscience 87: 463-477.

Winston SM, Hayward MD, Nestler EJ, Duman RS (1990). Chronic electroconvulsive seizures down-regulate expression of the immediate-early genes $\mathrm{c}$-fos and c-jun in rat cerebral cortex. J Neurochem 54: 1920-1925.

Woodbury DM, Woodbury JW (1990). Effects of vagal stimulation on experimentally induced seizures in rats. Epilepsia 31(Suppl 2): S7-S19.

Xu F, Gainetdinov RR, Wetsel WC, Jones SR, Bohn LM, Miller GW et al (2000). Mice lacking the norepinephrine transporter are supersensitive to psychostimulants. Nat Neurosci 3: 465-471.

Yousfi-Malki M, Puizillout JJ (1994). Induction of Fos-like protein in neurons of the medulla oblongata after electrical stimulation of the vagus nerve in anesthetized rabbit. Brain Res 635: 317-322.

Zhang W, Wang Z, Zhang W (2004). Characterizing blood pressure, heart rate, physical activity, circadian rhythm and their response to nos inhibitor and substrate in chf rats with telemetry. Vasc Dis Prev 1: 159-166. 